\title{
Advancing preparedness for clinical research during infectious disease epidemics
}

\author{
Nina Gobat (10) ${ }^{1}$ John Amuasi ${ }^{2}$, Yazdan Yazdanpanah ${ }^{3}$, Louise Sigfid ${ }^{4}$, \\ Hugh Davies ${ }^{5}$, John-Paul Byrne $\mathbb{1}^{6}$, Gail Carson ${ }^{4}$, Christopher Butler ${ }^{1}$, \\ Alistair Nichol $^{6}$ and Herman Goossens ${ }^{7}$
}

\begin{abstract}
Affiliations: ${ }^{1}$ Nuffield Dept of Primary Care Health Sciences, University of Oxford, Oxford, UK. ${ }^{2}$ Dept of Global Health, Kumasi Collaborative Center for Research in Tropical Medicine, Kumasi, Ghana. ${ }^{3}$ Dept Infectious Diseases, Hopital Bichat - Claude-Bernard, Paris, France. ${ }^{4}$ Centre for Tropical Medicine and Global Health, University of Oxford, Oxford, UK. ${ }^{5}$ Oxford Research Ethics Committee, Oxford, UK. ${ }^{6}$ Clinical Research Center, University College Dublin, Dublin, UK. ${ }^{7}$ Dept of Microbiology, University of Antwerp, Antwerp, Belgium.
\end{abstract}

Correspondence: Nina Gobat, Nuffield Dept of Primary Care Health Sciences, University of Oxford, Radcliffe Observatory Quarter, Woodstock Road, Oxford OX2 6CG, UK. E-mail: nina.gobatiphc.ox.ac.uk

@ERSpublications

Clinical research is vital for an effective response to infectious disease epidemics. To be viable, preparations must be made in anticipation of infectious disease epidemics and must address barriers to rapid deployment and implementation. bit.ly/2IzUlyv

Cite this article as: Gobat N, Amuasi J, Yazdanpanah Y et al. Advancing preparedness for clinical research during infectious disease epidemics. ERJ Open Res 2019; 5: 00227-2018 [https://doi.org/ 10.1183/23120541.00227-2018].

The Spanish Flu of 1918 reached every continent, infected one-third of the world's population and claimed over 50 million lives [1]. The centenary of this event presented a stark reminder of the threat of infectious disease epidemics to global health and security. These threats persist, fuelled in part by features of modern-day living such as climate change, globalisation, deforestation and population growth [2]. While progress has been made for managing infectious disease epidemics, critical gaps in leadership, governance, coordination and finance remain [3].

Patient-centred clinical research provides much needed evidence for clinical management and public health decisions in an infectious disease epidemic [4]. Building capability for clinical research is a core component of preparedness and the case for investment has been well made [5,6]. For many emerging infectious diseases, effective diagnostics, medications and vaccines are simply not available [7] and where these medical countermeasures are available, the evidence for their use in infectious disease epidemics is often limited. In many instances the point at which an epidemic occurs is the only time to gather data and generate new knowledge regarding disease characterisation, prevention and treatment. Experience from previous epidemics highlights how time and again, the research response is delayed and the narrow window of opportunity for enrolling patients during peak epidemic waves is missed. To be effective, research must be fast, flexible and integrated with the frontline response. However, research takes time to conceive, prepare, deliver and disseminate. Multisite, multi-country research responses are often needed to ensure sufficient patient numbers for conclusive results across demographics and research teams must navigate the requirements of multiple regional and national administrative and regulatory systems. Preparation must take place before and in anticipation of outbreaks and should address the multiple political, ethical, administrative, contractual, regulatory, logistic, economic and societal factors that influence the viability of conducting research in an outbreak. 
Members of the Global Research Collaboration for Infectious Disease Preparedness (GloPID-R) [8] have funded clinical research networks across multiple global regions, and in particular in sub-Saharan Africa. These networks have different models of operation adapted to different global regions and contexts, but are united by a common goal: to build capacity for delivering clinical research in response to infectious disease epidemics. The Platform for European Preparedness Against (Re-)emerging Epidemics (PREPARE) increases the ability for rapid clinical research response to emerging infectious disease outbreaks that are of public health concerns in Europe. In September 2018, these networks came together at a public meeting in Brussels coordinated by GloPID-R and PREPARE. The meeting aimed to share learning and solutions about how to overcome common bottlenecks when delivering research in this challenging context [9]. In total, 97 delegates from 24 countries attended the meeting, including infectious disease specialists, social scientists, microbiologists, field epidemiologists, public health specialists, regulators, policy makers and funders. Through expert-led presentations, workshops and discussion, delegates considered how progress can be made, particularly in three key areas (figure 1).

\section{Innovation in clinical research design and delivery}

The scientific, ethical and regulatory requirements of clinical research are such that many research activities are necessarily sequential: protocols must set out the design of research before a study can be approved; ethical and other regulatory bodies must confirm their approval before patients can be recruited patient or proxy consent must (usually) be obtained before patient data and samples can be collected; and data must be cleaned before it is analysed and findings disseminated. Rapid deployment of clinical studies requires innovations to research design, approval and delivery that reduce delay along this sequential process. For example, clinical research questions for likely candidate outbreaks can be pre-formulated: "hibernating" or "sleeping" studies can be pre- or partially approved [10]; mechanisms for expedited research governance review, using parallel approval processes, can be set out and rehearsed; lean research enrolment procedures with creative methods for exchanging information about research participation can be designed [11]; novel technology can be used for real-time collection of data; pre-approved clinical characterisation protocols can harmonise data capture and facilitate data sharing [12]; and research tasks can be aligned and embedded within routine practice to reduce burden on clinical teams.

The unique questions posed by infectious disease outbreaks call for innovation to the conception and design of clinical studies to provide answers that are pragmatic, workable and bolster public health responses. For example, studies orientated to enrolling patients with syndromic presentations of likely candidate infectious disease outbreaks during intra-epidemic periods would seamlessly continue enrolling patients at the point of an outbreak, and have the capacity to move rapidly from an observational to interventional mode. A suite of observational studies within the PREPARE consortium uses this approach to enrol patients with arthropod borne viral compatible febrile illness, sepsis-like-syndromes and acute respiratory infection [13]. Pragmatic and novel trial designs, such as Adaptive Platform Trials [14], offer promise.

FIGURE 1 Three areas for solutions to barriers that delay clinical research delivered during infectious disease outbreaks.

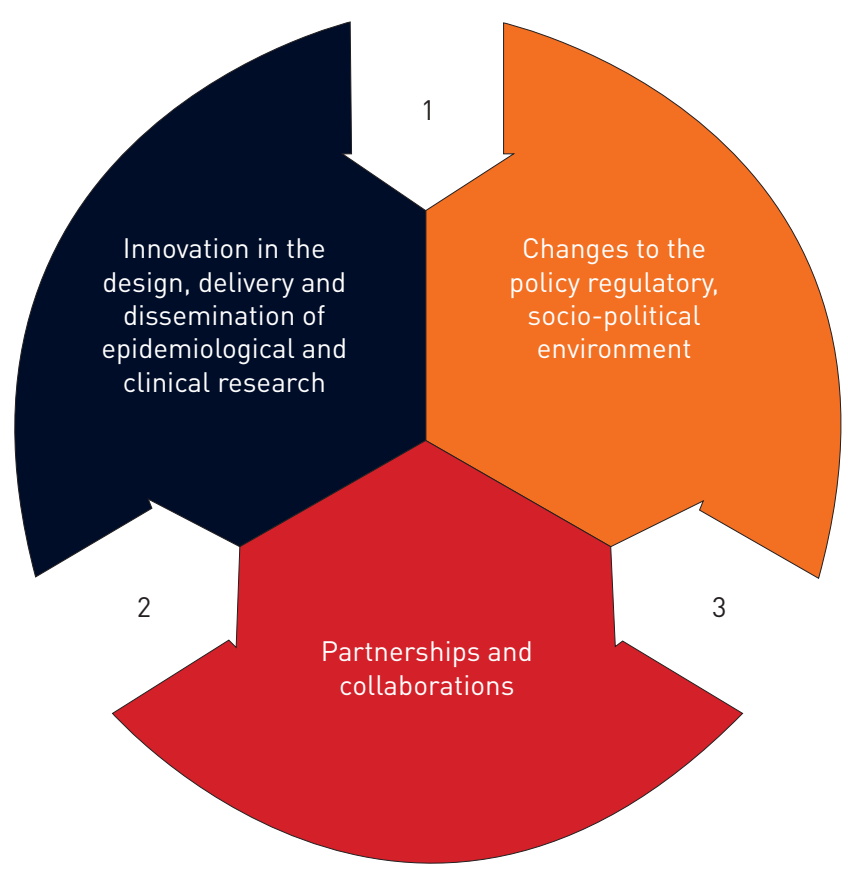


Examples include $\mathrm{ALIC}^{4} \mathrm{E}$, a pragmatic trial that has recruited over 3000 patients across 21 primary care networks in 15 European Union countries to evaluate the clinical and cost effectiveness of oseltamivir for patients with influenza-like illness in primary care $[15,16]$; and REMAP-CAP that evaluates treatment options for critically ill patients with community acquired pneumonia in Europe, Australia, New Zealand and Canada [17]. Interdisciplinary approaches are also key; for example, integrated social science research provides insight into multi-stakeholder understanding, and acceptability of research and can ensure contextually and culturally appropriate research features. Social science networks are now being funded to contribute to this work. Identifying ways to feedback outcomes of research, as well as considering wider questions about integration of research findings, are often neglected yet important areas of focus.

\section{An "enabling" regulatory, policy and socio-political environment}

Clinical research operates in a wider policy, regulatory and socio-political context. Key features of that context can operate as a barrier or an enabler to the delivery of clinical research during an ID outbreak. Preparedness for clinical research needs a strong policy context and needs to occur across sectors, professional disciplines and population groups. Processes and mechanisms for rapid response are needed among regulatory bodies, ethical review boards and funding communities. Progress has been made, for example, experience from the $2009 \mathrm{H} 1 \mathrm{~N} 1$ pandemic has led to pre- defined regulatory templates being developed for clinical trials of vaccines. These pre-prepared templates would expedite regulatory review for trials being deployed in a pandemic. However, preparedness initiatives need to move faster and further if they are to effectively support clinical research initiatives. A survey of Research Ethics Committees and National Competent Authorities across European Member states found that the majority of respondents did not know of, or did not have, expedited review processes, and those that knew of such processes, were not aware of the standard operating procedure to follow if required to expedite review [18]. Building regulatory capacity for effective review is also essential to ensure ethical standards are upheld when delivering clinical research in the operationally challenging circumstances of an epidemic [19]. Clinical research contracts and local site agreements often create the greatest time delay and pragmatic, sensible legal and administrative solutions are required. Socio-political trends signifying a rise of populism and anti-science movements also need to be actively countered to build an engaged, informed and receptive public to participate and benefit from infectious disease clinical research. Funding models that allow for nimble shifting of funds and importantly for sustainability of infectious disease research initiatives are needed to meet the unpredictable challenges of infectious disease clinical research preparedness. New models of research governance that promote and foster co-operation and collaboration are also essential.

\section{Partnerships and collaborations}

Infectious disease epidemic research preparedness requires strong interdisciplinary and cross-border collaborations with a clear focus on local capacity building and local leadership in epidemic hotspots. Strong partnerships are built on trust and commitment to a common purpose; and take time, both to build and sustain. However, there are multiple challenges to building equitable and mutually supportive partnerships. These include unequal access to funding, academic reward systems that incentivise competition over cooperation, silo-thinking reinforced by (artificial) disciplinary boundaries and commonly held assumptions regarding mutual capacities or needs. Fragmentation and competition among stakeholder groups, research initiatives and disciplines represent a lost opportunity for shared expertise and learning, and to strengthen global, national and regional research preparedness. A shared value base must ensure fairness and equity in opportunities, process, benefits, costs and outcomes [20]. Initiatives that stimulate organisational reflexivity are important, as are opportunities of knowledge exchange. The meeting resulted in strengthened partnerships across the clinical research networks and disciplines, including proposals for a cross-network advisory group working on mitigation of operational barriers in different global regions.

\section{Conclusion}

Being ready to deliver clinically useful research in the narrow timeframe available during an infectious disease epidemic requires pre-planning pre-positioning and practice [21] of research responses. However time and again this narrow window of opportunity is missed. The message from this international meeting of experts working to deliver a clinical research response to infectious disease outbreaks is clear: now is the time to act. We need to go further and faster with innovation and investment to harness the potential of science and shift the trajectory of infectious disease outbreaks protect the health of our populations and save lives.

Conflict of interest: N. Gobat reports grants from European Commission FP7, during the conduct of the study. J. Amusai has nothing to disclose Y. Yazdanpanah has been a board member receiving consultancy fees from ABBVIE, BMS, Gilead, MSD, Johnson \& Johnson, Pfizer and ViiV Healthcare in the past 4 years. L. Sigfid reports grants from 
European Commission FP7 and EU H2020, during the conduct of the study. H. Davies has nothing to disclose. J-P. Byrne reports grants from European Commission FP7, during the conduct of the study. G. Carson reports grants from European Commission H2020, during the conduct of the study. C. Butler reports grants from European Commission FP7, during the conduct of the study. A. Nichol reports grants from European Commission FP7, during the conduct of the study. H. Goossens reports grants from European Commission FP7, during the conduct of the study.

Support statement: This study received funding support from the European Commission: Horizon 2020 Framework Programme (643434) and the Seventh Framework Programme (602525). Funding information for this article has been deposited with the Crossref Funder Registry.

\section{References}

Taubenberger JK, Morens DM. 1918 influenza: the mother of all pandemics. Emerging Infect Dis 2006; 12: 15-22.

2 Smith KF, Goldberg M, Rosenthal S, et al. Global rise in human infectious disease outbreaks. J R Soc Interface 2014; 11: 20140950.

3 Leigh J, Moon S, Garcia E, et al. Is global capacity to manage outbreaks improving? An analysis. Geneva, Graduate Institute of International and Development Studies, 2018.

4 Rojek AM, Horby PW. Modernising epidemic science: enabling patient-centred research during epidemics. BMC Med 2016; 14: 212.

5 Commission on a Global Health Risk Framework for the Future. The neglected dimension of global security: a framework to counter infectious disease crises. 2016. http://nam.edu/GHRFreport

6 Lurie N, Manolio T, Patterson AP, et al. Research as a part of public health emergency response. N Engl J Med 2013; 368: 1251-1255.

7 Mehand MS, Al-Shorbaji F, Millett P, et al. The WHO R\&D Blueprint: 2018 review of emerging infectious diseases requiring urgent research and development efforts. Antiviral Res 2018; 159: 63-67.

8 Matthiessen L, Colli W, Delfraissy J-F, et al. Coordinating funding in public health emergencies. Lancet 2016; 387: 2197-2198.

9 Gobat N, Sigfrid L, Maskell K, et al. Reaching out: a meeting to advance clinical research preparedness for infectious disease outbreaks. 2018. www.prepare-europe.eu/Portals/0/Documents/Publications/Reaching\%20Out\%20Report_ PREPARE\%20GloPID-R_Final_2018\%2011\%2019.pdf?timestamp=15426439186968timestamp=1542643918696

10 Lim WS, Brittain C, Duley L, et al. Blinded randomised controlled trial of low-dose Adjuvant Steroids in Adults admitted to hospital with Pandemic influenza (ASAP): a trial "in hibernation", ready for rapid activation. Health Technol Assess 2015; 19: 1-78.

11 Gobat NH, Gal M, Butler CC, et al. Talking to the people that really matter about their participation in pandemic clinical research: a qualitative study in four European countries. Health Expect 2018; 21: 387-395.

12 Van Kerkhove MD, Reveiz L, Souza JP, et al. Harmonisation of Zika virus research protocols to address key public health concerns. Lancet Glob Health 2016; 4: e911-e912.

13 Platform for European Preparedness Against (Re-) emerging Epidemics. 3. Practice A: observational study workpackage 3. www.prepare-europe.eu/About-us/Workpackages/Workpackage-3

14 Berry SM, Connor JT, Lewis RJ. The platform trial: an efficient strategy for evaluating multiple treatments. JAMA 2015; 313: 1619-1620.

15 Butler CC, Coenen S, Saville BR, et al. A trial like ALIC ${ }^{4}$ : why design a platform, response-adaptive, open, randomised controlled trial of antivirals for influenza-like illness? ERJ Open Res 2018; 4: 00046-02018.

16 Bongard E, van der Velden AW, Cook J, et al. Antivirals for influenza-Like Illness? A randomised Controlled trial of Clinical and Cost effectiveness in primary CarE (ALIC4E): the ALIC4E protocol. BMJ Open 2018; 8: e021032.

17 Platform for European Preparedness Against (Re-) emerging Epidemics. 5. Practice C: Randomized, embedded multifactorial, adaptive platform trial severe CAP - workpackage 5. www.prepare-europe.eu/About-us/ Workpackages/Workpackage-5

18 Byrne J-P, Rahmani M, Davies H, et al. Pandemic research preparedness survey of national competent authorities and research ethics committees across Europe. Antwerp, PREPARE WP1, 2018.

19 ALERRT. "Ethics preparedness": Facilitating Ethics Review During Outbreaks. Recommendations arising from a joint ALERRT \& WHO workshop. 2018. www.alerrt.global/sites/www.alerrt.org/files/2018-06/alerrt_workshop_ recommendations_final_30may18_0.pdf

20 TDR Research Fairness Initiative report. Geneva, World Health Organization, 2018.

21 Webb SA, Nichol AD. Bending the pandemic curve: improving decision-making with clinical research. Crit Care Med 2018; 46: 442-446. 